\title{
On the $\mathrm{S}^{1}$-Segal Conjecture
}

By

\section{Goro NISHIDA*}

\section{§1. Introduction}

For a compact Lie group $G$ the Segal conjecture can be formulated similarly to that for finite groups as follows. Let $\pi_{k}^{G}\left(S^{0}\right)$ be the equivariant stable homotopy group [5]. Let $E G$ be a free contractible $G-C W$ complex and let $E G^{(r)}$ be the equivariant skeleton. The projection $E G^{(r)} \rightarrow *$ induces a homomorphism $\pi_{k}^{G}\left(S^{0}\right) \cong \pi_{G}^{-k}\left(S^{0}\right) \rightarrow \pi_{G}^{-k}\left(E G_{+}^{(r)}\right)$. It is well known that $\pi_{G}^{-k}\left(E G_{+}^{(r)}\right) \cong$ $\pi^{-k}\left(\left(E G^{(r)} / G\right)_{+}\right)$and we have a homomorphism $\alpha_{r}: \pi_{k}^{G(}\left(S^{0}\right) \rightarrow \pi^{-k}\left(\left(E G^{(r)} / G\right)_{+}\right)$. Since $\varliminf_{\longrightarrow} E G^{(r)} / G=B G$, we write $\varliminf_{1} \pi^{-k}\left(\left(E G^{(r)} / G\right)_{+}\right)$as $\mathscr{H}^{-k}(B G ; \boldsymbol{S})$, then we have a homomorphism

$$
\alpha: \pi_{k}^{G}\left(S^{0}\right) \longrightarrow \mathscr{H}^{-k}(B G ; S) .
$$

Note that if $G$ is not finite then $\mathscr{H}^{-k}(B G ; S)$ is not isomorphic to the actual stable cohomotopy group $\pi^{-k}\left(B G_{+}\right)$.

Let $A(G) \cong \pi_{0}^{G}\left(S^{0}\right)$ be the Burnside ring of $G$ defined by tom Dieck [5]. It is clear that $\alpha$ is continuous with respect to the $I(G)$-adic topology on $\pi_{k}^{G}\left(S^{0}\right)$ and the inverse limit topology on $\mathscr{H}^{-k}(B G ; \boldsymbol{S})$. Hence we have a continuous homomorphism

$$
\hat{\alpha}: \pi_{h}^{G}\left(S^{0} \hat{\imath}_{(G)} \longrightarrow \mathscr{H}^{-k}(B G ; S) .\right.
$$

If $G$ is finite then the solution of the Segal conjecture [4] asserts that $\hat{\alpha}$ is a topological isomorphism. But if $G$ is not finite then $\hat{\alpha}$ is seen to be not an isomorphism by a trivial reason. Let $G=S^{1}$, then $I\left(S^{1}\right)=0$ and the $I\left(S^{1}\right)$-adic completion is the identity. Let $k=1$, then by the tom Dieck splitting [6], $\pi_{1}^{S^{1}}\left(S^{0}\right)$ is a countable direct sum of $\boldsymbol{Z}$. On the other hand $\mathscr{H}^{-1}\left(B S^{1} ; S\right)$ is $\boldsymbol{Z} \oplus$ profinite group. Therefore those groups have different cardinalities. If $G=S^{1}$ and $k \leq 0$, then J. F. Adams [12] has announced that $\hat{\alpha}$ is an isomorphism. But even when $k=0$ the situation is still bad. For example let $G=O(2)$. Then $I(O(2))$ is a

* Department of Mathematics, Kyoto University, Kyoto 606, Japan. 
countable direct sum of $Z$. N. Minami has pointed out that $I(O(2))$-adic topology on $I(O(2))$ is the 2-adic topology. Therefore $I(O(2)) \hat{2}$ is not compact but $\tilde{\mathscr{H}}^{0}(B O(2) ; S)=\varliminf_{i m}\left\{B O(2)^{(r)}, S^{0}\right\}$ is compact. So the $I(G)$-adic topology is inadequate for compact Lie groups and the Segal conjecture for non finite groups should be stated as follows.

Conjecture. $\quad \alpha: \pi_{k}^{G}\left(S^{0}\right) \rightarrow \mathscr{H}^{-k}(B G ; S)$ has a dense image for $k \in Z$.

For $k \geq 0, \mathrm{M}$. Feshbach [13] has shown that the conjecture holds for any compact Lie group. Now the purpose of this paper is to prove the following.

Theorem. The Segal conjecture holds for $G=S^{1}$. Moreover $\alpha$ is an isomorphism if $k \leq 0$.

Our method is an approximation of $S^{1}$ by finite cyclic groups. For this we use the $S^{1}$-transfer and in Section 2 we explain this in more general situation. In Section 3 we show approximation theorems for stable cohomotopy and stable homotopy of $B S^{1}$, and in Section 4 the proof of the theorem will be given.

\section{§ 2. Compact Lie Group and Higher Transfer}

Let $G$ be a compact Lie group and $V$ a real $G$-module. We say that a closed $G$-manifold $M$ has a stable $V$-framing if there is a $G$-bundle monomorphism

$$
\varphi: V \oplus W \longrightarrow \tau_{G}(M) \oplus W
$$

onto a $G$-subbundle of $\tau_{G}(M) \oplus W$ for some $G$-module $W$ where $V=M \times V$ and $\tau_{G}(M)$ is the tangent $G$-bundle. Choose a $G$-invariant metric on $M$, then a $V$ framing determines a $G$-bundle $\alpha$ and a $G$-bundle isomorphism $\tau_{G}(M) \oplus W \cong$ $V \oplus W \oplus \alpha$. Choose a $G$-embedding $M \rightarrow U$ into a $G$-module $U$ and let $v$ be the normal bundle. If $U$ is large enough then the above isomorphism induces a $G$-bundle isomorphism $v \oplus \alpha \cong \boldsymbol{U}-\boldsymbol{V}$. Let $f$ be the composite

$$
U^{c} \stackrel{\gamma}{\longrightarrow} v^{c} \subset(v \oplus \alpha)^{c} \stackrel{\pi}{\longrightarrow}(U-V)^{c}
$$

where $\gamma$ is the Pontrjagin-Thom map and $\pi$ is the projection. We denote the stable class of $f$ by $\chi_{V}(M) \in \pi_{V}^{G}\left(S^{0}\right)$, the equivariant $V$-stem. $\quad \chi_{V}(M)$ depends only on the stable class of a $V$-framing. For $V=0, \pi_{0}^{G}\left(S^{0}\right)$ is identified with the Burnside ring $A(G)$ and clearly $\chi_{0}(M)=[M] \in A(G)$ in the sense of tom Dieck [5].

Let $H$ be a closed subgroup of $G$ and let $W_{H}=N_{G}(H) / H$. In [6] tom Dieck 
has shown that there is a homomorphism $\lambda_{H}: \pi_{n}^{W_{H}}\left(E W_{H+}\right) \rightarrow \pi_{n}^{G}\left(S^{0}\right)$ such that

$$
\lambda=\underset{(H)}{\oplus} \lambda_{H}: \underset{(H)}{\oplus} \pi_{n}^{W_{H}}\left(E W_{H+}\right) \longrightarrow \pi_{n}^{G}\left(S^{0}\right)
$$

is an isomorphism where $(H)$ runs through the conjugacy classes of subgroups of $G$. Let $M$ be an $n$-dim. free $W_{H}$-manifold with an $R^{n}$-framing. Then the Pontrjagin-Thom construction of the classifying $W_{H}$-map $M \rightarrow E W_{H}$ determines a class $[M] \in \pi_{n}^{W_{H}}\left(E W_{H+}\right)$. It is clear that the $G$-manifold $G \times_{N(H)} M$ has an $R^{n}$-framing induced from that of $M$. Then from the construction we easily see the following.

Lemma 2.1. $\lambda_{H}([M])=\chi_{R^{n}}\left(G \times_{N(H)} M\right) \in \pi_{n}^{G}\left(S^{0}\right)$.

Let now $F \stackrel{i}{\longrightarrow} E \stackrel{\pi}{\longrightarrow} B$ be a fibre bundle associated with a principal $G$-bundle $\tilde{E} \rightarrow B$. We suppose that $F$ is a closed $G$-manifold and $B$ is compact. Let $\tilde{\tau}$ be the tangent bundle along the fibre, i.e., $\tilde{\tau}=\tilde{E} \times{ }_{G} \tau_{G}(F)$. Let $\xi$ be a vector bundle over $B$. Then a stable map called a bundle transfer (Boardman [1])

$$
t: S B^{\xi} \longrightarrow S E^{\pi^{* \xi-\tau}}
$$

is defined by a similar way to the Becker-Gottlieb transfer [2]. Let now suppose that the fibre $F$ is $V$-framed so that $\tau_{G}(F) \widetilde{s} V \oplus \alpha$. Let $\tilde{\alpha}=\widetilde{E} \times{ }_{G} \alpha$ and $\tilde{V}=(\widetilde{E} \times$ $F \times V) / G$, then $\tilde{\alpha}-\tilde{\tau} \tilde{s}-\tilde{V}$. Let $\xi=0$, then composing $t$ with the canonical inclusion $E^{-\tilde{\tau}} \stackrel{j}{\longrightarrow} E^{-\tilde{\tau}+\tilde{\alpha}}$ we obtain a stable map

$$
t=t_{V}: S B^{0} \longrightarrow S E^{-\nabla}
$$

which is called a $V$-transfer. If $V=0$, then it is clearly the Becker-Gottlieb transfer.

Let $h^{*}$ be a multiplicative cohomology theory. Suppose that vector bundles $\xi$, $\tilde{\tau}$ and $\widetilde{V}$ are $h^{*}$-oriented. Then all stable bundles in the above construction are canonically $h^{*}$-oriented. Then via Thom isomorphisms $t$ and $t_{V}$ induce homomorphisms

$$
\pi_{!}: h^{i}(E) \longrightarrow h^{i-n}(B)
$$

and

$$
\pi_{V !}: h^{i}(E) \longrightarrow h^{i-d}(B)
$$

where $n=\operatorname{dim} F$ and $d=\operatorname{dim} V . \quad$ Note that $F$ is then $h^{*}$-oriented and let $[F] \epsilon$ $h^{n}(F)$ be the cohomology fundamental class.

Proposition 2.2. i) $\pi_{!}$is independent of $\xi$. 
ii) $\pi_{!}\left(x \cdot \pi^{*}(y)\right)=\pi_{!}(x) \cdot y \quad$ for $x \in h^{*}(E)$ and $y \in h^{*}(B)$.

iii) Suppose that there is an element $u \in h^{n}(E)$ such that $i^{*}(u)=[F], i^{*}$ : $h^{*}(E) \rightarrow h^{*}(F)$, then $\pi_{!}(u) \in h^{0}(B)$ is a unit.

iv) $\pi_{V !}(x)=\pi_{!}(\chi(\tilde{\alpha}) \cdot x), \chi(\tilde{\alpha}) \in h^{n-d}(E)$ is the Euler class of $\hat{\alpha}$.

Proof. i), ii) and iii) are obvious from [1] and iv) is clear from the fact that the composition $h^{*}(E) \stackrel{\cong}{\longrightarrow} \tilde{h}^{*}\left(E^{-\tilde{\tau}+\tilde{\alpha}}\right) \stackrel{j^{*}}{\longrightarrow} \tilde{h}^{*}\left(E^{-\tilde{\tau}}\right) \stackrel{\cong}{\longrightarrow} h^{*}(E)$ is just the multiplication with $\chi(\tilde{\alpha})$.

Let $H$ be a subgroup of $G$. Then we have fibre bundle $G / H \stackrel{i}{\longrightarrow} \widetilde{E} / H \stackrel{\pi}{\longrightarrow}$ $\tilde{E} / G=B$. Let $\operatorname{ad}(G)$ be the adjoint representation of $G$ on the tangent space $T(G)_{e}$ and let $\breve{\xi}=\widetilde{E} \times{ }_{G}$ ad $(G)$. Note that $\tau_{G}(G / H) \cong G \times{ }_{H}(\operatorname{ad}(G) / \operatorname{ad}(H))$ as $G$-vector bundles. Then we see that $\pi^{*} \tilde{\zeta}-\tilde{\tau} \cong \tilde{E} \times{ }_{H}$ ad $(H)$ and we have a bundle transfer $t: S\left(\widetilde{E}_{+} \wedge_{G}\right.$ ad $\left.(G)^{c}\right) \rightarrow S\left(\widetilde{F}_{+} \wedge_{H}\right.$ ad $\left.(H)^{c}\right)$ which is just the transfer of Becker-Schultz [3]. On the other hand let $d=d(G, H)=\operatorname{dim} W_{H}$. Then it is well known that $\operatorname{dim}(\operatorname{ad}(G) / \operatorname{ad}(H))^{H}=d$ and the inclusion $W_{H} \rightarrow G / H$ determines a canonical $R^{d}$-framing on $G / H$. Hence we have a transfer $t: S \Sigma^{d}\left(\tilde{E} / G_{+}\right)$ $\rightarrow S\left(\widetilde{E} / H_{+}\right)$. Let $\widetilde{E}=E G^{(r)}$ and using the naturality of the transfer we can take a limit and obtain stable maps

$$
t=t_{\mathrm{ad}}: S\left(E G_{+} \wedge_{G} \operatorname{ad}(G)^{c}\right) \longrightarrow S\left(E H_{+} \wedge_{H} \operatorname{ad}(H)^{c}\right)
$$

and

$$
t=t_{G / H}: S \Sigma^{d}\left(B G_{+}\right) \longrightarrow S\left(B H_{+}\right)
$$

which will be called a $G / H$-transfer. Let $K$ be a subgroup of $H$. Then in general $t_{H / K^{\circ}} \Sigma^{d(H, K)} t_{G / H} \neq t_{G / K}$, but if $G$ is abelian the equality clearly holds.

In [7] Hauschild has shown that there is an isomorphism

$$
\mu=\mu_{G}: \pi_{k}\left(E G_{+} \wedge_{G} \operatorname{ad}(G)^{c}\right) \longrightarrow \pi_{k}^{G}\left(E G_{+}\right) .
$$

Then from the construction we easily see the following

Lemma 2.3. The following diagram is commutative

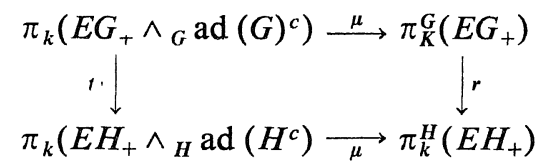

where $r$ is the homomorphism given by restricting the G-action. 


\section{§3. Approximation by Cyclic Groups}

Let $X$ be a connected CW-complex and let $p$ be a prime number. Let $X_{p}$ denote the $p$-adic completion of $X$ of Sullivan [10]. Let $F$ be a connected $H$ space such that $\pi_{i}(F)$ is a finite $p$-group for any $i$. Then by the obstruction theory we easily see that the natural map

$$
\left[X_{p}^{\wedge}, F\right] \longrightarrow[X, F]
$$

is an isomorphism. Let $\left\{X_{\lambda}\right\}_{i \in \Lambda}$ be a direct system of finite CW-complexes with a countable index set $\Lambda$. Let $X=\operatorname{hocolim} X_{\lambda}$. Let $\boldsymbol{E}$ be a connected locally finite spectrum. We put

$$
\tilde{\mathscr{H}}^{i}\left(X ; \boldsymbol{E}_{p}^{\hat{p}}\right)=\lim _{i}\left(\tilde{h}^{i}\left(X_{\lambda} ; \boldsymbol{E}\right) \otimes \hat{\boldsymbol{Z}}_{p}\right)
$$

where $h^{i}\left(X_{\lambda} ; \boldsymbol{E}\right)$ is the generalized cohomology theory defined by $\boldsymbol{E}$. Let $\left\{X_{\lambda}\right\} \rightarrow\left\{Y_{\mu}\right\}$ be a morphism of direct systems and let $f: X \rightarrow Y$ be the induced map. Then we obtain an induced homomorphism

$$
f^{*} ; \widetilde{\mathscr{H}}^{i}\left(Y ; \boldsymbol{E}_{p}^{\wedge}\right) \longrightarrow \widetilde{\mathscr{H}}^{i}\left(X ; \boldsymbol{E}_{p}^{\wedge}\right) .
$$

Let $\boldsymbol{E} \rightarrow \boldsymbol{F} \rightarrow \boldsymbol{G}$ be a cofibration of spectra. Note that $\tilde{h}^{i}\left(X_{\lambda} ; \boldsymbol{E}\right) \otimes \boldsymbol{Z}_{p}^{\hat{p}}$ is a compact topological group. Hence there is no $\varliminf^{1}$ and we obtain an exact sequence

$$
\longrightarrow \mathscr{H}^{i}\left(X ; \boldsymbol{E}_{p}^{\hat{p}}\right) \longrightarrow \mathscr{H}^{i}\left(X ; \boldsymbol{F}_{p}^{\hat{p}}\right) \longrightarrow \mathscr{H}^{i}\left(X ; \boldsymbol{G}_{p}\right) \longrightarrow .
$$

Let $Z_{p^{r}} \subset S^{1}$ be the standard inclusion. Let $Z_{p^{\text {ec }}}=\lim _{\underline{\eta}} \boldsymbol{Z}_{p^{r}}$, then we have an inclusion $\boldsymbol{Z}_{p^{\infty}} \subset S^{1}$. Note that it is factored as $\boldsymbol{Z}_{p^{\infty}} \subset \boldsymbol{Q} / \boldsymbol{Z} \subset S^{1}$. Those inclusions induce maps $B Z_{p^{\infty}} \rightarrow B(\boldsymbol{Q} / \boldsymbol{Z}) \rightarrow B S^{1}$ which are all denoted by $j . \quad$ It is well known [10] that

$$
\hat{j_{p}}:\left(B Z_{p^{\infty}}\right)_{p} \longrightarrow\left(B S^{1}\right)_{p}
$$

and

$$
j^{\wedge}:(B(\boldsymbol{Q} / \boldsymbol{Z}))^{\wedge} \longrightarrow\left(B S^{1}\right)^{\wedge}
$$

are homotopy equivalences, where ()$^{\wedge}$ is the profinite completion. We have $B S^{1}=\varliminf_{\left(B S^{1}\right)^{(n)}}$ and $B Z_{p^{\infty}}=\varliminf_{(}\left(B Z_{p^{n}}\right)^{(n)}$ and the map $j: B Z_{p^{\infty}} \rightarrow B S^{1}$ is clearly filtrated. Let $S$ be the sphere spectrum.

Proposition 3.1. For any prime $p$ and any integer $i$, the homomorphism $j^{*}: \widetilde{\mathscr{H}}^{i}\left(B S^{1} ; \boldsymbol{S}_{p}^{\wedge}\right) \rightarrow \widetilde{\mathscr{H}}^{i}\left(B \boldsymbol{Z}_{p^{\infty}} ; \boldsymbol{S}_{p}\right)$ is an isomorphism. 
Proof. Let $\tilde{S} \rightarrow \boldsymbol{S} \rightarrow \boldsymbol{K}(\boldsymbol{Z})$ be the 0-connective fibration of the sphere spectrum. Then we have a commutative diagram

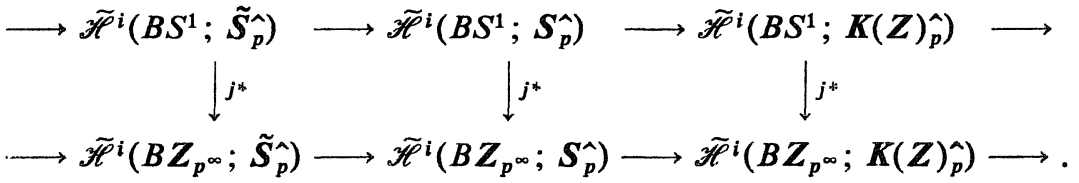

First note that

$$
\begin{aligned}
j^{*} ; \tilde{\mathscr{H}}^{i}\left(B S^{1} ; K(Z) \hat{p}\right) & \cong H^{i}\left(B S^{1} ; \hat{Z}_{p}\right) \rightarrow \widetilde{\mathscr{H}}^{i}\left(B Z_{p^{\infty}} ; K(Z)_{p}\right) \\
& \left.\cong \varliminf\left(H^{i}(B) Z_{p^{r}} ; Z_{p}^{\hat{p}}\right)\right)
\end{aligned}
$$

is an isomorphism. Next for given $i$ choose $l \geq 0$ such that $l+i \geq 0$. Then one easily see that

$$
\widetilde{\mathscr{H}}^{i}\left(X ; \tilde{\boldsymbol{S}}_{p}^{\wedge}\right) \cong\left[\Sigma^{l}\left(X_{+}\right),\left(\widetilde{Q S}^{l+i}\right)_{p}\right]
$$

where $\widetilde{Q S}^{l+i}$ is the $l+i$ connective fibre space of $Q S^{l+i}$. Note that $\pi_{j}\left(\left(\widetilde{Q S^{l+i}}\right)_{p}\right)$ is a finite $p$-group for any $j$. Then since $\hat{j_{p}}:\left(B Z_{p^{\infty}}\right)_{p} \rightarrow\left(B S^{1}\right)_{p}$ is a homotopy equivalence we see that

$$
j^{*}: \tilde{\mathscr{H}}^{i}\left(B S^{1}: \tilde{\boldsymbol{S}_{p}}\right) \longrightarrow \widetilde{\mathscr{P}}^{i}\left(B Z_{p^{\infty}}: \tilde{\boldsymbol{S}_{p}}\right)
$$

is an isomorphism. Hence the proposition follows from the five lemma.

Now consider the commutative diagram

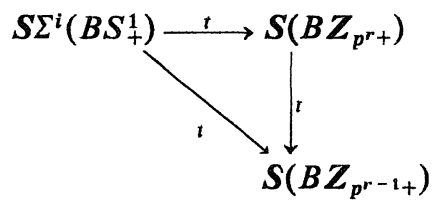

of the transfer maps associated with $Z_{p^{r-1}} \subset Z_{p^{r}} \subset S^{1}$. Then we have a homomorphism

$$
\varliminf_{t^{*}}: \pi_{i}\left(S \Sigma^{1}\left(B S_{+}^{1}\right)\right) \longrightarrow \varliminf_{i}\left(S\left(B Z_{p^{r+}}\right)\right) .
$$

Proposition 3.2. $\varliminf^{*}$ is a p-adic complection.

Proof. From the cofibration $S^{0} \rightarrow B Z_{p^{r+}} \rightarrow B Z_{p^{r}}$ we obtain an inverse system of cofibrations $\left\{\boldsymbol{S}^{0}\right\}_{r} \rightarrow\left\{\boldsymbol{S}\left(B \boldsymbol{Z}_{p^{r_{+}}}\right)\right\}_{r} \rightarrow\left\{\boldsymbol{S}\left(B \boldsymbol{Z}_{p^{r}}\right)\right\}_{r}$. Note that $\left\{\boldsymbol{S}^{0}\right\}_{r}$ is $S^{0} \stackrel{p}{\longleftarrow} S^{0} \stackrel{p}{\longleftarrow} \cdots$. Then we easily see that $\prod_{i}\left(S\left(B Z_{p^{r+}}\right)\right) \cong \varliminf \pi_{i}\left(S B Z_{p^{r}}\right)$. Let $\left(S \Sigma^{1}\left(B S_{+}^{1}\right)\right)_{p}^{\hat{p}}$ be the $p$-adic completion of the spectrum $\boldsymbol{X}=\boldsymbol{S} \Sigma^{1}\left(B S_{+}^{1}\right)$. Let $f: X \rightarrow F$ be a spectra map where $F$ is a connective $C W$-spectrum such that $\pi_{i}(\boldsymbol{F})$ is a finite $p$-group for any $i$. Then $\boldsymbol{X}_{p}^{\wedge}$ can be given as a functorial inverse 
limit $\varliminf_{i m} \boldsymbol{F}$. We are then enough to show that $\left\{\boldsymbol{X} \stackrel{t}{\longrightarrow} S B Z_{p^{r}}\right\}_{r}$ is cofinal in $\{\boldsymbol{X} \rightarrow \boldsymbol{F}\}$. Let $\pi: B Z_{p^{r}} \rightarrow B S^{1}$ be the projection. Then $S^{1} / Z_{p^{r}}$-transfer $t$ induces a homomorphism

$$
\pi_{1 !}: H^{i}\left(B Z_{p^{r}} ; Z_{p}\right) \longrightarrow H^{i-1}\left(B S^{1} ; Z_{p}\right) .
$$

By Proposition 2.2, iii) we see that $\pi_{1 !}$ is an isomorphism if $i$ is odd. Then one easily see that

$$
\varliminf^{*}: \varliminf^{i} \tilde{H}^{i}\left(B Z_{p^{r}} ; Z_{p}\right) \longrightarrow \tilde{H}^{i}\left(\Sigma^{1}\left(B S_{+}^{1}\right) ; Z_{p}\right)
$$

is an isomorphism for any $i$. Then by the obstruction theory (Postnikov system) similar to Sullivan [10], we see that $\left\{S \Sigma^{1}\left(B S_{+}^{1}\right) \stackrel{t}{\longrightarrow} S B Z_{p^{r}}\right\}$ is cofinal.

\section{§4. Proof of the Theorem}

Let $Z_{p^{r-1}} \subset Z_{p^{r}} \subset S^{1}$ be the standard inclusions. Consider the commutative diagram of the restriction homomorphisms

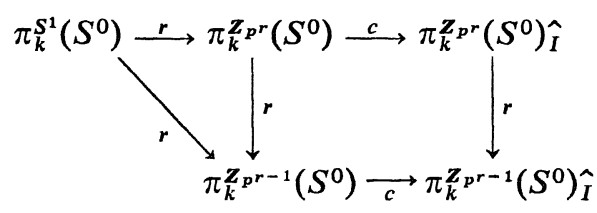

where ()$\hat{I}$ is the $I(G)$-adic completion. Then we have a homomorphism

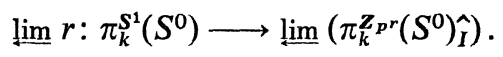

By the Milnor exact sequence for $B Z_{p^{\infty}}=\varliminf_{p^{r}} B Z_{p^{r}}$ we see that the canonical map

$$
\omega: h^{-k}\left(B \boldsymbol{Z}_{p^{\infty}} ; \boldsymbol{S}\right) \longrightarrow \varliminf^{-k}\left(B \boldsymbol{Z}_{p^{r}}: \boldsymbol{S}\right)
$$

is an isomorphism for any $k$. For the reduced groups we see that $\tilde{h}^{-k}\left(B Z_{p^{\infty}} ; \boldsymbol{S}\right)=\tilde{\mathscr{H}}^{-k}\left(B Z_{p^{\infty}} ; S\right) \rightarrow \widetilde{\mathscr{H}}^{-k}\left(B Z_{p^{\infty}} ; \boldsymbol{S}_{p}^{\wedge}\right)$ is an isomorphism. Then we have a commutative diagram

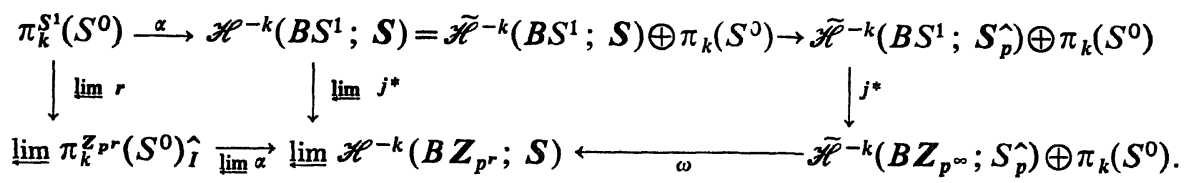

By the above argument $\omega$ is an isomorphism. By Proposition 3.1, $j^{*}$ is an isomorphism. By the solution of the Segal conjecture for cyclic groups [9], we see 
that $\varliminf_{\alpha} \alpha$ is an isomorphism.

Now according to $k$, the proof is devided into two cases. First suppose that $k<0$. Then by the tom Dieck splitting, $\pi_{k}^{S^{1}}\left(S^{0}\right) \cong \pi_{k}^{Z}{ }^{r}\left(S^{0}\right) \cong 0$. Hence we see that $\widetilde{\mathscr{P}}^{-k}\left(B S^{1} ; \boldsymbol{S}_{p}\right)=0$ for any $p$. Then we easily see that $\widetilde{\mathscr{H}}^{-k}\left(B S^{1} ; S\right)$ $=0$ and hence $\tilde{\mathscr{H}}^{-k}\left(B S^{1} ; S\right)=0$. This shows that $\alpha$ is an isomorphism.

Next suppose that $k \geq 0$. Let $H$ be a subgroup of $S^{1}$ and let $H_{r}=H \cap Z_{p^{r}}$. Then $Z_{p^{r}} / H_{r} \subset S^{1} / H$ and we have the transfer $t: S \Sigma^{1}\left(B\left(S^{1} / H\right)_{+} \rightarrow S\left(B\left(Z_{p^{r}} / H_{r}\right)_{+}\right)\right.$. By Lemmas 2.1 and 2.3 we have a commutative diagram

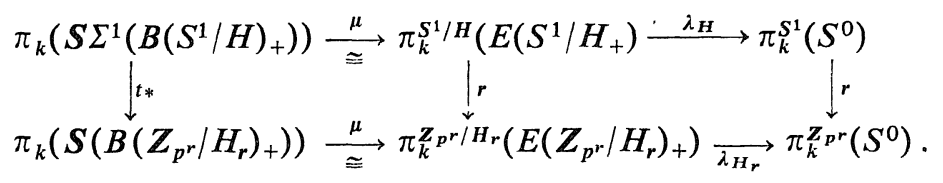

Let $\tilde{\pi}_{k}^{S^{1}}\left(S^{0}\right)=\operatorname{Coker}\left[\lambda_{S^{1}} ; \pi_{k}\left(S^{0}\right) \rightarrow \pi_{k}^{S^{1}}\left(S^{0}\right)\right]$ and similarly for $\tilde{\pi}_{k}^{Z^{r}}\left(S^{0}\right)$. Then we have the restriction homomorphism $r: \tilde{\pi}_{k}^{S^{1}}\left(S^{0}\right) \rightarrow \tilde{\pi}_{k}^{Z_{p} r}\left(S^{0}\right)$. Consider the diagram

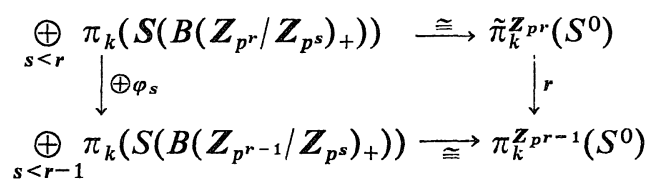

where $\varphi_{r-1}=0$ and $\varphi_{s}=t_{*}$ if $s<r-1$ and $t: S\left(B Z_{p^{r-s_{+}}}\right) \rightarrow \boldsymbol{S}\left(B \boldsymbol{Z}_{p^{r-s-1+}}\right)$ is the transfer. Then clearly the above diagram is commutative. Then from the following commutative diagram

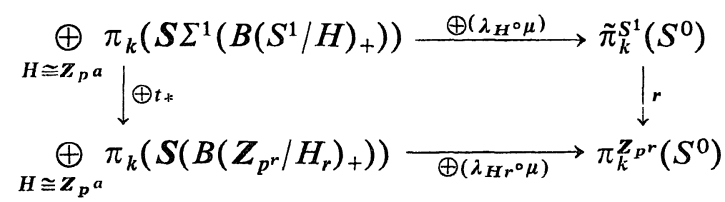

we obtain a commutative diagram

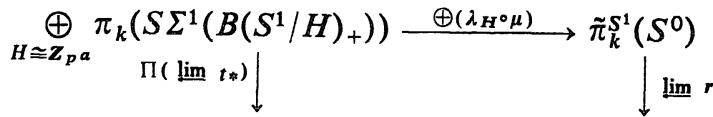

$$
\begin{aligned}
& \prod_{H \cong Z_{p a}} \prod_{k}\left(S\left(B\left(Z_{p^{r}} / H_{r}\right)_{+}\right)\right) \longrightarrow \varliminf_{\pi} \tilde{\pi}_{k}^{Z_{p r}}\left(S^{0}\right) \text {. }
\end{aligned}
$$

Note that $H_{r}=\mathbb{Z}_{p^{r}} \cap \boldsymbol{Z}_{p^{a}}$ and $\varliminf_{p^{r}} / H_{r}=\boldsymbol{Z}_{p^{\infty}} / \boldsymbol{Z}_{p^{a}} \cong \boldsymbol{Z}_{p^{\infty}}$. Hence by Propo-

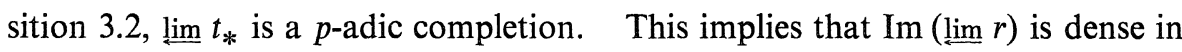
$\varliminf \tilde{\pi}_{k}^{Z_{p} r}\left(S^{0}\right)$, and hence so is for $\varliminf_{r} r: \pi_{k}^{S^{1}}\left(S^{0}\right) \rightarrow \prod_{k}^{Z} \pi_{p r}\left(S^{0}\right)$. Let $k=0$, then $\tilde{\pi}_{k}^{S^{1}}\left(S^{0}\right)=0$ and hence $\varliminf_{0}^{\boldsymbol{Z}^{p}}\left(S^{0}\right)=\varliminf A\left(\boldsymbol{Z}_{p^{r}}\right) \cong \boldsymbol{Z}$. This clearly implies that 
$\varliminf_{A} A\left(\boldsymbol{Z}_{p^{r}}\right)_{I} \cong \boldsymbol{Z}$. Then $\alpha: \boldsymbol{Z} \rightarrow \boldsymbol{Z}$ is clearly an isomorphism. Finally let $k>0$. Then $\pi_{k}^{Z_{p^{r}}}\left(S^{0}\right)$ is a finite group and hence the canonical map $\pi_{k}^{Z_{p} r}\left(S^{0}\right) \rightarrow \pi_{k}^{Z_{p r}}\left(S^{0}\right) \hat{I}$ is an epimorphism. Hence so is $\varliminf_{k} \pi_{k}^{Z^{r}}\left(S^{0}\right) \rightarrow \varliminf \pi_{k}^{Z_{p r}}\left(S^{0}\right)_{I}$. Then from the diagram $(D)$ we see that $\operatorname{Im} \alpha$ is dense. This completes the proof.

As a remark we state the structure of the actual stable cohomotopy group $\tilde{h}^{k}\left(B S^{1} ; S\right)=\left\{\Sigma^{-k} B S^{1}, S^{0}\right\}$ for $k \geq 0$.

Proposition 4.1. Let $k \geq 0$, then $\tilde{h}^{k}\left(B S^{1} ; S\right) \cong 0$ if $k$ is even or $k=1$, and $\cong$ $\hat{Z} / \boldsymbol{Z}$ if $k=2 i+1, i>0$.

Proof. If $k$ is even then $\lim ^{1} \tilde{h}^{k-1}\left(\left(B S^{1}\right)^{(r)} ; S\right)=0$ and the result follows from the main theorem. Next consider the following commutative diagram

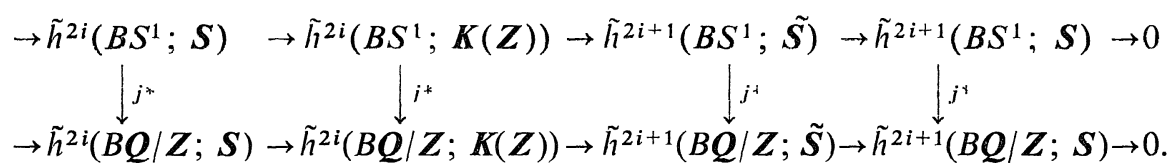

By Proposition 3.1 we see that $j^{*}: \tilde{h}^{*}\left(B S^{1} ; \tilde{\boldsymbol{S}}\right) \rightarrow \tilde{h}^{*}(B \boldsymbol{Q} / \boldsymbol{Z} ; \tilde{\boldsymbol{S}})$ is an isomorphism. Note that there is no $\varliminf^{1}$ for $B \boldsymbol{Q} / \boldsymbol{Z}$. Then by the Segal conjecture for cyclic groups we see that $\tilde{h}^{i}(B \boldsymbol{Q} / \boldsymbol{Z} ; \boldsymbol{S})=0$ for $i>0$. Then from the above diagram we immediately see that $\tilde{h}^{-1}\left(B S^{1} ; S\right)=0$, and $\tilde{h}^{2 i+1}\left(B S^{1} ; S\right) \cong$ Coker $\left[j^{*}: \tilde{h}^{2 i}\left(B S^{1} ; \boldsymbol{K}(\boldsymbol{Z})\right) \rightarrow \tilde{h}^{2 i}(B \boldsymbol{Q} / \boldsymbol{Z} ; \boldsymbol{K}(\boldsymbol{Z}))\right] \cong \boldsymbol{Z} / \tilde{\boldsymbol{Z}}$ if $i>0$.

\section{References}

[1] Boardman, J. M., Stable homotopy theory, Chapter V-Duality and Thom spectra, mimeographed, University of Warwick, 1966.

[2] Becker, J. C. and Gottlieb, D. H., The transfer map and fibre bundles, Topology, 14 (1975), 1-12.

[3] Becker, J.C. and Schultz, R. E., Equivariant function spaces and stable homotopy theory I, Comment. Math. Helv., 49 (1974), 1-34.

[4] Carlsson, G., Equivariant stable homotopy and Segal's Burnside ring conjecture, preprint.

[5] tom Dieck, T., Transformation groups and representation theory, Lecture Notes in Math. 766, Springer-Verlag.

[6] - Orbittypen und äquivariante Homologie II, Arch. Math., 215 (1975), 235-250.

[7] Hauschild, H., Zerspaltung äquivarianter Homotopiemengen, Math. Ann., 230 (1977), 279-292.

[8] Nishida, G., The transfer homomorphism in equivariant generalized cohomology theories, J. Math. Kyoto Univ., 18 (1978), 435-451.

[9] Ravenel, D., The Segal conjecture for cyclic groups, Bull. London Math. Soc., 13 (1981), 42-44. 
[10] Sullivan, D., Genetics of homotopy theory and the Adams conjecture, Ann. of Math., 100 (1974), 1-79.

[11] Wirthmüller, K., Equivariant homology and duality, Manuscripta Math., 11 (1974), 373-390.

[12] Adams, J. F., Graem Segal's Burnside ring conjecture, Bull. Amer. Math. Soc., 6 (1982), 201-212.

[13] Feshbach, M., The cohomology of classifying spaces of compact Lie groups and their finite subgroups; The Segal conjecture for compact Lie groups, to appear. 